\title{
Caracterización Socioeconómica del Sistema de Producción de Papa en la Provincia de Barranca
}

\author{
Socioeconomic Characterization the Production System \\ Potato in the Province of Barranca
}

\author{
Sergio Contreras Liza', Sady García Bendezú
}

\begin{abstract}
RESUMEN
Objetivo: caracterizar socioeconómicamente las fincas productoras de papa en 3 localidades de la provincia de Barranca. Métodos: Para ello se realizó un muestreo aleatorio considerando 3 distritos de las zonas productoras de papa dentro de este ámbito: Supe, Barranca y Pativilca, tomándosela información de 67 fincas en 14 puntos de muestreo. La encuesta estuvo constituida por 30 preguntas considerando factores económicos, sociales y ambientales de la producción de papa. Los datos tomados de las encuestas fueron procesados estadísticamente mediante el programa Infostat, para fines de clasificación y sistematización de la información, utilizándose el análisis de varianza no paramétrico para una vía de clasificación de Kruskal y Wallis a un nivel de confianza de $95 \%$. Resultados: El rendimiento promedio de papa en Barranca fue 28,25 t.ha $^{-1}$ mientras que los costos de producción alcanzaron un valor promedio 19000 nuevos soles. El tamaño de la finca de papa en la provincia fue 5,54 has en promedio; dichas fincas están conducidas por productores con un bajo nivel de inserción en organizaciones sociales, con acceso a los servicios básicos y bajo un sistema agrario en el que predomina el monocultivo y la baja diversidad en especies y de cultivares de papa. Conclusiones: Se mostraron diferencias estadísticas en las tres localidades de producción de la provincia de Barranca en los indicadores de naturaleza económica y ambiental, pero menor variabilidad en indicadores sociales.
\end{abstract}

Palabras Clave: Sustentabilidad, indicadores socioeconómicos, agroecosistemas, Perú.

\begin{abstract}
Objective: The aim of the research was to characterize socioeconomically potato farms in 3 locations in the province of Barranca: Supe, Barranca and Pativilca. Methods: A random sampling for 3 districts of potato producing areas in this province was conducted taking the information of 67 farms in 14 sampling sites. The survey consisted of 30 questions considering economic, social and environmental potato production factors. The data from the surveys were processed statistically by Infostat program for the purpose of classification and systematization of information, using nonparametric analysis of variance for one-way classification of Kruskal and Wallis at a confidence level of $95 \%$. Results: The average yield of potatoes was $28,25 \mathrm{t}^{\text {. ha }}{ }^{-1}$ while production costs averaged value 19000 soles $(\$ 5429,0)$. The size of the potato farm in the province was 5.54 hectares in average; these farms are conducted by producers with low insertion in social organizations, with access to basic services and under an agricultural system in which predominates monocultures and low diversity of species and cultivars of potato. Conclusion: Statistical differences were foundfor economic and environmental indicators between three locationsat the province of Barranca, but less variability was detectedfor social indicators.
\end{abstract}

Keywords: Sustainability, socioeconomic indicators, agro-ecosystems, Peru.

\footnotetext{
${ }^{1}$ Docente. Facultad de Ciencias Agrarias, Industriales Alimentarias y Ambiental. Universidad Nacional José Faustino Sánchez Carrión. Lima - Perú

${ }^{2}$ Director del Laboratorio de Suelos. Universidad Nacional Agraria La Molina. Lima - Perú.
} 


\section{INTRODUCCIÓN}

En la región Lima provincias prevalece el sistema de parcelas agrícolas originadas como consecuencia de las desmembración de las ex Cooperativas Agrarias de Producción (CAPs) establecidas en la Reforma Agraria de la década de 1970, siendo aún hoy el modelo económico predominante de organización campesina. Las parcelas o fundos son las unidades productivas básicas de la agricultura en la región Costa y son conducidas por agricultores que utilizan mayormente tecnología agrícola intensiva y hacen por lo general uso de capital financiero. Un fenómeno resaltante en la región es la aparición hace dos décadas de la modalidad de arrendamiento de los terrenos de cultivo o parcelas agrícolas para la siembra de papa, páprika, maíz entre otros cultivos; muchos de estos nuevos arrendatarios proceden del sector comercial agropecuario de la Sierra y Lima.

La papa es cultivada en más de 300 mil hectáreas a nivel nacional (Eguren, 2012). El rendimiento promedio nacional es de 13,4 t.ha $^{-1}$.; más del $80 \%$ del área dedicada a la papa es pequeña agricultura, con superficie menor a las 20 hectáreas (MINAGRI, 2013). Se conoce que el cultivo es muy sensible a una serie de estreses ambientales bióticos y abióticos; estos problemas, aunados al hecho que la especie se propaga comercialmente por medios vegetativos hacen que la sanidad del cultivo sea un problema considerable para la producción de material de siembra de calidad y para la producción comercial (FAO, 2008).

En la región Lima se cultivaron alrededor de 6266 has de papa durante la campaña agrícola 2013-2014 con un rendimiento medio de 23,7 t.ha ${ }^{-1}$, según datos de la Oficina Regional de Promoción Agraria de Lima (MINAGRI, 2015). En este sistema de producción de papa se utiliza tecnología agrícola como aplicación intensiva de insumos agrícolas (particularmente fertilizantes y agroquímicos), riego, control fitosanitario, asistencia técnica además de otros factores socioeconómicos que es necesario ponderar, para evaluar si son usados en forma racional y de acuerdo a los estándares de sustentabilidad ambiental, económica y social. Los mayores rendimientos de papa blanca se obtienen en las zonas de Costa, donde se utiliza una tecnología de cultivo más moderna que incluye sistemas de riego tecnificado. La mayoría de las zonas de producción de papa blanca que se comercializa en Lima Metropolitana, están articuladas logísticamente a los mercados (Devaux et al., 2010).

Las metodologías denominadas de "investigación de sistemas prediales o de finca" enfatizan la comprensión de los sistemas agrícolas tradicionales como punto de partida, evalúan los antecedentes de la zona, realizan exámenes en el terreno que incluyen entrevistas a los agricultores respecto a las características del predio y analizan acerca del porqué los agricultores emplean métodos particulares de producción (Altieri, 1997). Para Masera, Astier y López Ridaura (1999) el concepto de sustentabilidad se define a partir de cinco atributos generales de los agroecosistemas o sistemas de manejo: (a) productividad; (b) estabilidad, confiabilidad y resiliencia; (c) adaptabilidad; (d) equidad; (e) auto-dependencia.

El presente trabajo de investigación tuvo como objetivo caracterizar socioeconómicamente las fincas productoras de papa en 3 localidades de la provincia de Barranca, para luego hacer propuestas alternativas de gestión para optimizar el sistema de producción de papa en la provincia de Barranca, región Lima.

\section{MATERIALES Y MÉTODOS}

El estudio comprendió la descripción y diagnóstico de las fincas con cultivos de papa en 3 zonas agroecológicas consideradas de Barranca y la evaluación de las propiedades específicas que determinan su comportamiento dinámico, limitaciones y potencialidades.

1. Caracterización de los predios agrícolas de Barranca: La población objetivo estuvo constituida por los agricultores productores de papa en la provincia de Barranca, región Lima con una superficie total estimada de siembra de papa de 1940 has. (MINAGRI, 2015). Se efectuó un muestreo aleatorio considerando 3 distritos de las zonas productoras de papa dentro de este ámbito: Supe, Barranca y Pativilca; en total se tomaron 14 puntos de muestreo o zonas de producción en los 3 distritos de la provincia. El tamaño de muestra se calculó aplicando la fórmula de Scheaffer et al. (1987). En total se entrevistó a 67 agricultores conductores de predios agrícolas cubriendo un área muestreada de 336 hectáreas sembradas con papa en el año 2015. Se desarrolló un cuestionario de 30 preguntas tomando en cuenta factores económicos, sociales y ambientales de la producción de papa de las fincas en la provincia. Para el diseño de la encuesta, se tuvo en cuenta la opinión de expertos del Programa de Papa y del Departamento de Economía de la Universidad Nacional Agraria La Molina. Antes de llevar a cabo las entrevistas, se validó el cuestionario realizando un sondeo en la localidad con 10 productores de papa para ajustar algunos criterios de clasificación. Las variables evaluadas fueron (basadas en la metodología de Sarandon, 2002):

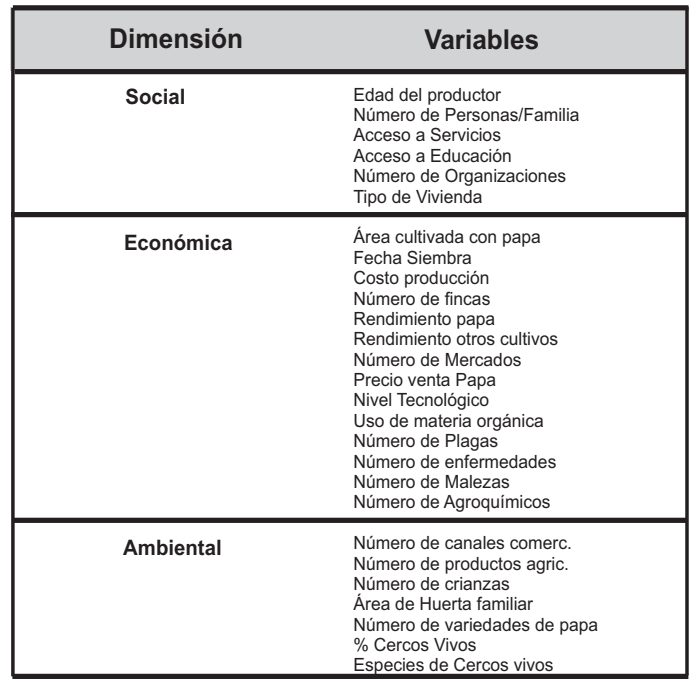

Big Bang Faustiniano 2016; 5(2): 37 - 41 
2. Análisis de la información: Para fines del estudio se hizo uso complementariamente de los siguientes recursos de información: Hojas de cálculo, Mapas cartográficos, Estadísticas del INEI, Cartillas de evaluación, Datos meteorológicos, Análisis de Suelos, Data de ensayos agronómicos, Publicaciones científicas y Tesis. Los datos tomados de las encuestas fueron inicialmente registrados en hojas de cálculo y luego procesados estadísticamente mediante el programa Infostat, para fines de clasificación y sistematización de la información, utilizándose el análisis de varianza no paramétrico para una vía de clasificación de Kruskal y Wallis. Finalmente, se elaboraron tablas y gráficos para la presentación, descripción y análisis de la información.

\section{RESULTADOS Y DISCUSIÓN}

En la tabla 1, se puede observar las áreas sembradas con papa en la región Lima, donde destaca la provincia de Barranca con más del 30\% del área sembrada a nivel regional; de acuerdo a INEI (2013) el área cultivada con papa en la región Lima según datos del IV CENAGRO alcanzó las 7213 has lo que supone una reducción del cultivo de papa en la Costa central, hecho mayormente atribuido a la presencia anunciada del fenómeno del Niño (ENFEN, 2015) y a sus posibles efectos en la tuberización y producción de la papa (Egúsquiza, 2008).

Tabla 1. Superficie sembrada con papa en 3 provincias de la Región-Lima.

\begin{tabular}{lcc}
\hline Provincia & Superficie estimada 2015 & \% Región Lima \\
\hline Barranca & 1940 & 31,23 \\
Huaral & 1526 & 24,57 \\
Cañete & 952 & 15,33 \\
Sub-Total & 4418 & $71,13 \%$ \\
Otras provincias & 1793 & $28,87 \%$ \\
\hline Total Lima & 6211 & $100,0 \%$ \\
\hline
\end{tabular}

En la tabla 2, se presentan los valores promedios de las principales variables evaluadas en la encuesta en las tres dimensiones señaladas, advirtiendo que en el nivel social la edad promedio del productor de papa era 47 años, con 4-5 personas dependientes en promedio y con acceso al menos a cinco servicios básicos; la mayoría de las fincas registraba ausencia de servicios de saneamiento. El tamaño promedio de las fincas fue seis hectáreas, aunque el área dedicada a papa era algo menor (5 has.), lo que coincide con la información del INEI (2013); los productores de papa en Barranca no están organizados o su nivel de afiliación a organizaciones es incipiente.

Tabla 2. Características socioeconómicas de los productores de papa en la provincia de Barranca: valores medios de los indicadores.

\begin{tabular}{rlcccl}
\hline $\mathbf{N}^{\circ}$ & Variable & Media & & & Unidades \\
\hline 1 & Edad & 47,2 & $+/-$ & 12,29 & años \\
2 & N Personas/Fam. & 4,4 & $+/-$ & 1,46 & \#personas \\
3 & Acceso a servicios & 5,03 & $+/-$ & 1,27 & \#servicios \\
4 & Área Finca & 6,21 & $+/-$ & 4,1 & has. \\
5 & Organización & 0,73 & $+/-$ & 0,59 & \#organizac. \\
6 & Área papa & 5,02 & $+/-$ & 3,54 & has. \\
\hline 7 & Fecha Siembra papa & 28,3 & $+/-$ & 4,2 & semanas \\
\hline 8 & Costo producción & 19067 & $+/-$ & 3543 & S/.ha' \\
9 & Núm. fincas & 1,43 & $+/-$ & 1,23 & \#fincas \\
10 & Rendto. Papa & 28,25 & $+/-$ & 5,26 & t.ha-1 \\
11 & Rendto. Otro Cult. & 10,64 & $+/-$ & 3,11 & t.ha \\
12 & Mercados & 1 & $+/-$ & 0 & \#mercados \\
13 & Precio venta Papa & 0,78 & $+/-$ & 0,18 & S/..'-1 \\
14 & Nivel. Técnico & 6,18 & $+/-$ & 0,94 & \#factores \\
15 & Uso mat. Orgánico & 7,66 & $+/-$ & 9,31 & t.ha \\
16 & Núm. Plagas & 2,18 & $+/-$ & 0,9 & \#especies \\
17 & Núm. Enfermedades & 1,16 & $+/-$ & 0,54 & \#especies \\
18 & Núm. Malezas & 0,62 & $+/-$ & 0,91 & \#especies \\
19 & Núm. Agroquímicos & 4,86 & $+/-$ & 1,55 & \#productos \\
20 & Núm. Canal Común & 1,03 & $+/-$ & 0,17 & \#canales \\
21 & Núm. productos & 1,51 & $+/-$ & 0,79 & \#cultivos \\
22 & Núm. Crianzas & 0,73 & $+/-$ & 0,96 & \#crianzas \\
23 & Área Huerta & 0,04 & $+/-$ & 0,15 & has. \\
24 & Núm. variedades & 1,75 & $+/-$ & 0,68 & \#cultivares \\
25 & $\%$ Cercos Vivos & 25,08 & $+/-$ & 32,02 & \%fincas \\
26 & Núm. Especies & 0,88 & $+/-$ & 1,16 & \#especies \\
\hline & & & & & \\
\hline
\end{tabular}

La tabla 3 nos muestra que en los indicadores sociales evaluados, no existió variabilidad atribuida a las tres localidades de siembra para estos indicadores a un nivel de confianza del $95 \%$; ello sugiere que en el caso de edad del productor, acceso a servicios básicos, nivel de inserción en organizaciones y tamaño de la finca los valores de los indicadores nos muestran una homogeneidad de las condiciones sociales del cultivo de papa en la provincia de Barranca.

En relación a los aspectos técnico-económicos, las fechas de siembra de papa fluctuaron alrededor de la semana 28 (segunda semana de julio) aunque hubieron siembras desde inicios del mes de junio a finales de agosto; ello está en relación al periodo vegetativo de cultivo, la disponibilidad de semilla y las condiciones climáticas prevalecientes, que inciden en la tuberización y en la presencia de plagas y enfermedades (Egúsquiza, 2008).

Por otro lado, los costos de producción por hectárea bordearon los 19000 nuevos soles y el rendimiento de tubérculos fue algo superior a las 28 t. ha $^{-1}$, lo que hace un costo unitario por kilo de papa producido de 0,68 centavos.

Tabla 3. Análisis no paramétrico de la variabilidad para indicadores sociales en 3 localidades productoras de papa de la provincia de Barranca.

\begin{tabular}{|c|c|c|c|c|c|c|}
\hline Variable & Distrito & Medias & DE & Median & H & $\mathrm{p}$ \\
\hline $\begin{array}{l}\text { Número de } \\
\text { Organizaciones }\end{array}$ & $\begin{array}{l}\text { Barranca } \\
\text { Pativilca } \\
\text { Supe }\end{array}$ & $\begin{array}{l}0.75 \\
0.79 \\
0.67\end{array}$ & $\begin{array}{l}0.74 \\
0.42 \\
0.56\end{array}$ & $\begin{array}{l}1 \\
1 \\
1\end{array}$ & 0.49 & 0.72 \\
\hline Área de la Finca & $\begin{array}{l}\text { Barranca } \\
\text { Pativilca } \\
\text { Supe }\end{array}$ & $\begin{array}{l}6.78 \\
6.14 \\
5.67\end{array}$ & $\begin{array}{l}4.05 \\
5 \\
3,47\end{array}$ & $\begin{array}{l}6 \\
4 \\
5\end{array}$ & 1.58 & 0.45 \\
\hline $\begin{array}{l}\text { Acceso a } \\
\text { Servicios }\end{array}$ & $\begin{array}{l}\text { Barranca } \\
\text { Pativilca } \\
\text { Supe }\end{array}$ & $\begin{array}{l}4.88 \\
5.16 \\
5.08 \\
\end{array}$ & $\begin{array}{l}0.85 \\
1.61 \\
1.35\end{array}$ & $\begin{array}{l}5 \\
5 \\
5\end{array}$ & 1.41 & 0.47 \\
\hline Edad & $\begin{array}{l}\text { Barranca } \\
\text { Pativilca } \\
\text { Supe }\end{array}$ & $\begin{array}{l}47.1 \\
51.61 \\
43.68\end{array}$ & $\begin{array}{l}15.06 \\
10.47 \\
10.04\end{array}$ & $\begin{array}{l}46 \\
49 \\
42 \\
\end{array}$ & 4.81 & 0.09 \\
\hline
\end{tabular}

*Valores de $p<0,05$ son estadísticamente significativos para la prueba de Kruskall-Wallis 
Asimismo en la tabla 4 se advierte que las variables fechas de siembra, incidencia de plagas y de malezas, nivel tecnológico usado, rendimiento por hectárea y precio de venta en chacra mostraron diferencias significativas entre localidades, lo que puede indicar distintos patrones o sistemas de cultivo entre localidades para estos parámetros. También las variables costos de producción, tamaño de la finca, incidencia de enfermedades y uso de agroquímicos no mostraron variabilidad significativa entre las localidades muestreadas, por lo que se puede asumir que en estos indicadores existe un nivel de homogeneidad. Al respecto, Altieri (1997) menciona que la complejidad de los agroecosistemas está íntimamente vinculada al reconocimiento de que existe una gran heterogeneidad ecológica y/o cultural, lo que se ha observado en el caso de los productores de papa en Barranca cuyos indicadores económicos y ambientales han mostrado diferencias entre localidades.

Tabla 4. Análisis no paramétrico de la variabilidad de indicadores económicos en 3 localidades productoras de papa de la provincia de Barranca.

\begin{tabular}{|c|c|c|c|c|c|c|}
\hline Variable & Distrito & Medias & DE & Median & H & $p$ \\
\hline $\begin{array}{l}\text { Costo de } \\
\text { producción } \\
\text { papa, S/.ha }\end{array}$ & $\begin{array}{l}\text { Barranca } \\
\text { Pativilca } \\
\text { Supe }\end{array}$ & $\begin{array}{l}19979 \\
18789 \\
18375\end{array}$ & $\begin{array}{l}4103 \\
3687 \\
2667\end{array}$ & $\begin{array}{l}20000 \\
19000 \\
19000\end{array}$ & 2.21 & 0.33 \\
\hline $\begin{array}{c}\text { Fecha de } \\
\text { Siembra papa, } \\
\text { semanas }\end{array}$ & $\begin{array}{l}\text { Barranca } \\
\text { Pativilca } \\
\text { Supe }\end{array}$ & $\begin{array}{l}27.3 \\
30.3 \\
27.7\end{array}$ & $\begin{array}{l}4.13 \\
4.07 \\
3.97\end{array}$ & $\begin{array}{l}27 \\
31 \\
28\end{array}$ & 6.79 & 0.033 \\
\hline $\begin{array}{l}\text { Tamaño de finca } \\
\text { papa, has }\end{array}$ & $\begin{array}{l}\text { ca Barranca } \\
\text { Pativilca } \\
\text { Supe }\end{array}$ & $\begin{array}{l}4.9 \\
5.7 \\
4.6\end{array}$ & $\begin{array}{l}2.09 \\
4.87 \\
3.55\end{array}$ & $\begin{array}{l}5 \\
4 \\
4\end{array}$ & 1.98 & 0.37 \\
\hline $\begin{array}{c}\text { Uso de } \\
\text { Agroquimicos }\end{array}$ & $\begin{array}{l}\text { Barranca } \\
\text { Pativilca } \\
\text { Supe }\end{array}$ & $\begin{array}{l}4.7 \\
4.8 \\
5.1\end{array}$ & $\begin{array}{l}1.6 \\
1.1 \\
1.8\end{array}$ & $\begin{array}{l}4 \\
5 \\
5\end{array}$ & 0.97 & 0.6 \\
\hline $\begin{array}{l}\text { Incidencias de } \\
\text { Malezas }\end{array}$ & $\begin{array}{l}\text { Barranca } \\
\text { Pativilca } \\
\text { Supe }\end{array}$ & $\begin{array}{l}0.56 \\
1.26 \\
0.10\end{array}$ & $\begin{array}{l}0.86 \\
0.99 \\
0.44\end{array}$ & $\begin{array}{l}0 \\
2 \\
0\end{array}$ & 10.76 & $\underset{*}{0.0003}$ \\
\hline $\begin{array}{l}\text { Incidencias de } \\
\text { Enfermedades }\end{array}$ & $\begin{array}{l}\text { Barranca } \\
\text { Pativilca } \\
\text { Supe }\end{array}$ & $\begin{array}{l}1.25 \\
1.05 \\
1.17 \\
\end{array}$ & $\begin{array}{l}0.53 \\
0.4 \\
0.64 \\
\end{array}$ & $\begin{array}{l}1 \\
1 \\
1 \\
\end{array}$ & 1.04 & 0.46 \\
\hline $\begin{array}{l}\text { Incidencia de } \\
\text { Plagas }\end{array}$ & $\begin{array}{l}\text { Barranca } \\
\text { Pativilca } \\
\text { Supe }\end{array}$ & $\begin{array}{l}1.79 \\
2.11 \\
2.63 \\
\end{array}$ & $\begin{array}{l}0.66 \\
0.74 \\
1.06\end{array}$ & $\begin{array}{l}2 \\
2 \\
3\end{array}$ & 8.94 & $\underset{*}{0.006}$ \\
\hline $\begin{array}{c}\text { Nivel } \\
\text { Tecnológico }\end{array}$ & $\begin{array}{l}\text { Barranca } \\
\text { Pativilca } \\
\text { Supe }\end{array}$ & $\begin{array}{l}5.88 \\
5.63 \\
6.92\end{array}$ & $\begin{array}{l}0.95 \\
0.68 \\
0.58\end{array}$ & $\begin{array}{l}6 \\
6 \\
7\end{array}$ & 22.00 & $\underset{*}{0.0000}$ \\
\hline $\begin{array}{c}\text { Precio venta } \\
\text { Papa, S/. }\end{array}$ & $\begin{array}{l}\text { Barranca } \\
\text { Pativilca } \\
\text { Supe }\end{array}$ & $\begin{array}{l}0.68 \\
0.89 \\
0.78\end{array}$ & $\begin{array}{l}0.19 \\
0.07 \\
0.17\end{array}$ & $\begin{array}{l}0.8 \\
0.9 \\
0.8\end{array}$ & 16.73 & $\underset{*}{0.0002}$ \\
\hline $\begin{array}{l}\text { Rendimiento } \\
\text { Papa, t.ha }\end{array}$ & $\begin{array}{l}\text { Barranca } \\
\text { Pativilca } \\
\text { Supe }\end{array}$ & $\begin{array}{l}30.42 \\
23.05 \\
30.21\end{array}$ & $\begin{array}{l}4.68 \\
2.72 \\
4.51\end{array}$ & $\begin{array}{l}30 \\
23 \\
30\end{array}$ & 28.61 & $\underset{*}{0.0000}$ \\
\hline
\end{tabular}

Finalmente en el caso de los indicadores ambientales, se muestra en la tabla 5 que existió variabilidad entre localidades para número de variedades de papa utilizadas aunque predominó el monocultivo de un solo genotipo en Barranca y Pativilca con el cultivar Canchan y recientemente también con el cultivar Única. En el caso de diversidad de productos en la finca, solo en la localidad de Supe se presentó el caso de presencia de más de 1 producto durante el ciclo del cultivo de papa, por ser esta zona de siembra de hortalizas y pan llevar para los mercados locales. En el resto de indicadores ambientales como presencia de cercos vivos y área para la producción familiar, no existieron diferencias entre localidades, teniendo estas variables valores relativamente bajos.
Tabla 5. Análisis no paramétrico de la variabilidad de indicadores ambientales en 3 localidades productoras de papa de la provincia de Barranca.

\begin{tabular}{|c|c|c|c|c|c|c|}
\hline Variable & Distrito & Medias & $\mathrm{DE}^{+}$ & Median & H & $p$ \\
\hline $\begin{array}{l}\text { Número de } \\
\text { Especies de } \\
\text { Cerco }\end{array}$ & $\begin{array}{l}\text { Barranca } \\
\text { Pativilca } \\
\text { Supe }\end{array}$ & $\begin{array}{l}0.65 \\
0.58 \\
1.33\end{array}$ & $\begin{array}{l}1.03 \\
0.51 \\
1.49\end{array}$ & $\begin{array}{l}0 \\
1 \\
1\end{array}$ & 2.97 & 0.17 \\
\hline $\begin{array}{l}\text { \%Cercos vivos } \\
\text { en la finca }\end{array}$ & $\begin{array}{l}\text { Barranca } \\
\text { Pativilca } \\
\text { Supe }\end{array}$ & $\begin{array}{l}23.9 \\
26.6 \\
25.0\end{array}$ & $\begin{array}{l}39.51 \\
26.57 \\
29.12 \\
\end{array}$ & $\begin{array}{l}0 \\
20 \\
18 \\
\end{array}$ & 1.20 & 0.5 \\
\hline $\begin{array}{c}\text { Número de } \\
\text { Variedades de } \\
\text { papa }\end{array}$ & $\begin{array}{l}\text { Barranca } \\
\text { Pativilca } \\
\text { Supe }\end{array}$ & $\begin{array}{l}1.54 \\
2.32 \\
1.50\end{array}$ & $\begin{array}{l}0.51 \\
0.67 \\
0.59\end{array}$ & $\begin{array}{l}2 \\
2 \\
1\end{array}$ & 13.92 & $\underset{*}{0.0002}$ \\
\hline $\begin{array}{l}\text { Área de Huerta } \\
\text { Familiar }\end{array}$ & $\begin{array}{l}\text { Barranca } \\
\text { Pativilca } \\
\text { Supe }\end{array}$ & $\begin{array}{l}0.09 \\
0.00 \\
0.03\end{array}$ & $\begin{array}{l}0.24 \\
0.00 \\
0.08\end{array}$ & $\begin{array}{l}0 \\
0 \\
0\end{array}$ & 1.12 & 0.15 \\
\hline $\begin{array}{c}\text { Número de } \\
\text { productos en la } \\
\text { finca }\end{array}$ & $\begin{array}{l}\text { Barranca } \\
\text { a Pativilca } \\
\text { Supe }\end{array}$ & $\begin{array}{l}1.63 \\
1.11 \\
1.71\end{array}$ & $\begin{array}{l}0.82 \\
0.32 \\
0.91\end{array}$ & $\begin{array}{l}1 \\
1 \\
2\end{array}$ & 6.09 & $\underset{*}{0.016}$ \\
\hline
\end{tabular}

"Valores de $p<0,05$ son estadísticamente significativos para la prueba de Kruskall- Wallis

`DE: Desviación estándar de la media.

\section{CONCLUSIÓN}

En general, los sistemas de producción de papa en las tres localidades elegidas de la provincia de Barranca, mostraron diferencias dependientes de la localidad y de las condiciones de producción imperantes en la provincia. El rendimiento promedio de papa en Barranca fue 28,25 t.ha ${ }^{-1}$ mientras que los costos de producción alcanzaron un valor promedio 19000 nuevos soles. El tamaño de la finca de papa en la provincia fue 5,54 has en promedio; dichas fincas son conducidas por productores con un bajo nivel de inserción en organizaciones sociales, con acceso a los servicios básicos y bajo un sistema agrario en el que predomina el monocultivo y la baja diversidad en especies y de cultivares de papa.

\section{AGRADECIMIENTO}

La investigación fue financiada con fondos del Canon Regional y del Gas de Camisea (FOCAM) promovidos por el Vicerrectorado de Investigación de la Universidad Nacional José Faustino Sánchez CarriónHuacho, que permitió la ejecución del proyecto durante el 2014-2015. Se agradece asimismo, al siguiente personal administrativo de la citada Universidad: Amalia Magdalena Gonzales Marín, Yuri Gamonal Abanto, Adolfo Fausto Figueroa Rosadio, Rossana Emperatriz Garrido Oyola y María Julia Fernández Curay. Asimismo, se reconoce la colaboración y asesoría del Dr. Sady García Bendezú del Laboratorio de Suelos de la UNALM, del Ing. MSc. Rolando Egúsquiza Bayona, fitomejorador del cultivo de papa y docentes del Departamento de Economía y Planificación de la UNALM. 


\section{REFERENCIAS BIBLIOGRÁFICAS}

Altieri, M.A. 1997. Enfoque Agroecológico para el Desarrollo de Sistemas de Producción Sostenibles en los Andes. Ed. CIED. Lima-Perú. $92 \mathrm{p}$.

Banco Mundial. 2008. Informe sobre el desarrollo mundial. Agricultura para el desarrollo. Banco Mundial. Parte I, II y III.

Devaux, A.; Ordinola, M; Hibon, A; Flores, R. 2010. El sector papa en la región andina: Diagnóstico y elementos para una visión estratégica (Bolivia, Ecuador y Perú). Centro Internacional de la Papa. Lima. 386 p.

Eguren, F. 2012. Eficiencia y rendimientos en la agricultura peruana. La revista Agraria No. 141: 11.

Egúzquiza, R.2008. La Papa. Editorial Librería Técnica Agropecuaria Distribuidora S.A. Lima.

ENFEN. 2015. Informe técnico ENFEN. Comité Multisectorial encargado del Estudio Nacional del Fenómeno El Niño (ENFEN). Año $1 \mathrm{~N}^{\circ} 8$. Noviembre de 2015.

FAO .1990. El enfoque de sistemas agropecuarios para el desarrollo SAD. Servicio de Administración de Unidades Agropecuarias y Producción Económica-FAO. P. 13-34.

INEI. 2013. Resultados Definitivos. IV Censo Nacional Agropecuario 2012. Lima. Julio 2013. 63 pp.

MINAGRI. 2013. Principales Aspectos Agroeconómicos de la Cadena Productiva de Papa. Ministerio de Agricultura y Riego. Lima. 42 pp.
MINAGRI. 2015. Intenciones de siembra 2015-2016. DGESEP. Ministerio de Agricultura y Riego. Lima. Julio 2015.

Masera, O.; Astier, M. \& López-Ridaura, S. 1999. Sustentabilidad y manejo de recursos naturales. El Marco de Evaluación MESMIS. GIRA.A.C. México. 109 p.

Oswald, A.; Calvo P, D.; Zúñiga D. \& Arcos J. 2010.Evaluating soil rhizobacteria for their ability to enhance plant growth and tuber yield in potato. Ann ApplBiol157: 259-271

Quijandría, B. 1994. Aspectos teóricos y metodológicos del sistema y de la unidad de producción. Conferencia. Lima-Perú. p. 34-43.

Sarandon, S.J.; Flores, CC. 2014. Agroecología: bases teóricas para el diseño y manejo de agroecosistemas sustentables. 1a ed. La Plata: Universidad Nacional de La Plata. 467 P.

Sarandon, S. J. 1998. The development and use of sustainability indicators: a need for organic agriculture evaluation. XII International Scientific Conference IFOAM 1998. 16/19 Noviembre 1998, Mar del Plata, Argentina: pp. 135.

Sarandon, S. J. 2002. El desarrollo y uso de indicadores para evaluar la sustentabilidad de los agroecosistemas. En Santiago J. Sarandon (editor): Agroecología. El camino hacia una agricultura sustentable. Ediciones Científicas Americanas Capítulo 20: 393-414.

Scheaffer, R.; Mendenhall, W. \& Ott, L. 1987. Elementos de muestreo. Editorial Iberoamericana. México DF. 309 p. 